\title{
Geometrical Interpretation of Singular Value Decomposition(Svd) \& Applications of SVD
}

\author{
Jajimogga Raghavendar \\ Research Scholar \\ Department of Mathematics \\ Rayalaseema University \\ Kurnool, Andhra Pradesh, India \\ jm_raghu@yahoo.co.in
}

\author{
V.Dharmaiah \\ Retd. Professor \\ Department of Mathematics, \\ Osmania University \\ Hyderabad, Telangana, India
}

\begin{abstract}
The SVD is familiar toipc in Linear Algebra and it is a powerful techinque in many matrix computations and analysis. This paper is a dicussion of the geometric structure of a matrix using SVD. In SVD matrix can be transformed from one vector space to another vector space. The SVD has fundamental importance in several differnet applications of Linear Algebra.
\end{abstract}

Keywords: SVD, Geometric Structure of a Matrix, Vector Space,

\section{INTRODUCTION}

Singular value decomposition

For any $m \times m$ matrix A, the following decomposition always exist

$$
\begin{gathered}
A=U D V^{T}, A \in R^{m \times m} \\
U^{T} U=U U^{T}=I_{m}, \in R^{m \times m} \\
V^{T} V=V V^{T}=I_{m}, V \in R^{n \times n}
\end{gathered}
$$

$\therefore U, V$ are two orthogonal matrices and $D \in R^{m \times n}$ Diagonal matrices with non- negative entries of the diagonal called singular values.

If rank of $\mathrm{A}$ is $r<(m, n)$ the SVD can represented in reduced form

$$
\begin{gathered}
A=U D V^{T}, A \in R^{m \times n} \\
U \in R^{m \times r} \\
V \in R^{n \times r} \\
D \in R^{r \times r}
\end{gathered}
$$

Let $A=U D V^{T}=\sum_{i=1}^{r} D_{i i} U_{i} V_{i}^{T}$

This $m \times n$ matrix $U_{i} V_{i}^{T}$ is the product of column vector $U_{i}$ and the transpose of column vector $V_{i}$. It has rank.Thus $\mathrm{A}$ is weighted summation of $\mathrm{r}$ rank matrices

\section{GEOMETRIC INTERPRETATION OF THE SVD}

The SVD has a nice, simple geometric interpretation. It is very easy to draw in 2-Dimension.

Let $U=\left[\begin{array}{ll}u_{1} & u_{2}\end{array}\right]$ and $V^{T}=\left[\begin{array}{l}v_{1}^{T} \\ v_{2}^{T}\end{array}\right]$ 

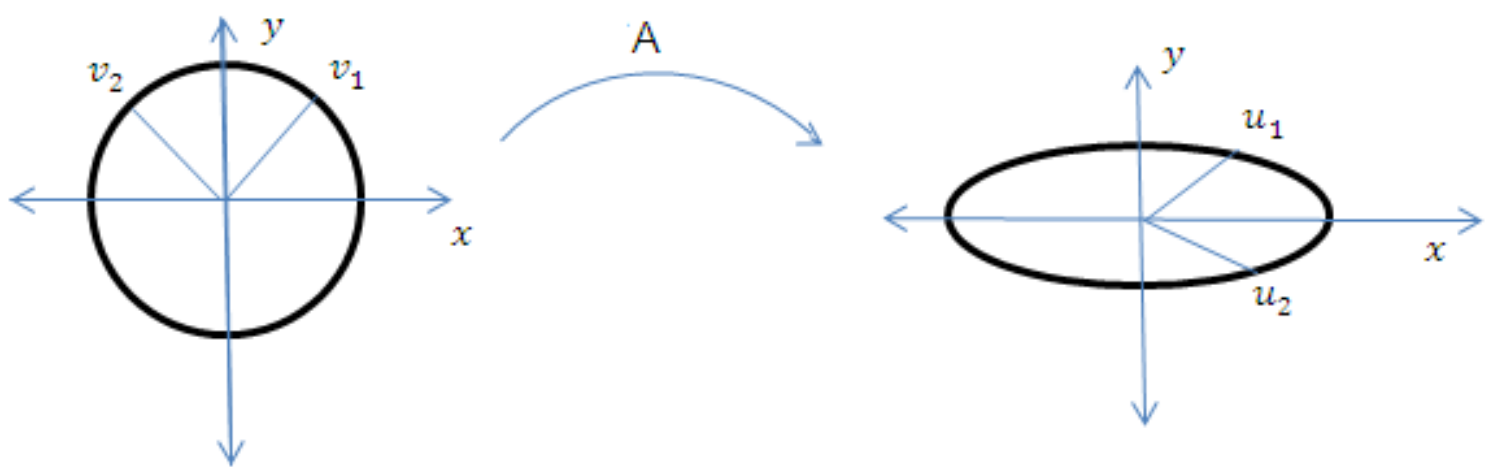

Let $A=U D V^{T}$

$$
\begin{gathered}
A V=\left(U D V^{T}\right) V \\
A V=U D\left(V^{T} V\right) \\
A V=U D I \\
A V=U D
\end{gathered}
$$

Let us take the unit circle and linear transformed by A, we get an ellipse. The pre-factor of D is $\mathrm{U}$, $u_{1} \quad u_{2}$ are the major and minor axis of the ellipse. The post factor of $\mathrm{D}$ is $V^{T}$. The vector $v_{1}, v_{2}$ in $V^{T}$ are the vectors that get mapped to the major and the minor axis. In 3-D the sphere is transformed to ellipsoid.

Another way to say sum of this is the set $A v_{1}=\sigma_{i} u_{i}$

Which you can see by this 2-D example

$$
\begin{gathered}
{\left[\begin{array}{c}
V_{1}^{T} \\
v_{2}^{T}
\end{array}\right] v_{1}=\left[\begin{array}{l}
1 \\
0
\end{array}\right]} \\
U D\left[\begin{array}{l}
V_{1}^{T} \\
v_{2}^{T}
\end{array}\right] v_{1}=U D \\
\left(U D V^{T}\right) V_{1}=U D\left[\begin{array}{l}
1 \\
0
\end{array}\right] \\
A v_{1}=U D\left[\begin{array}{l}
1 \\
0
\end{array}\right] \\
\therefore U D\left[\begin{array}{l}
1 \\
0
\end{array}\right]=\sigma_{i} u_{i}
\end{gathered}
$$

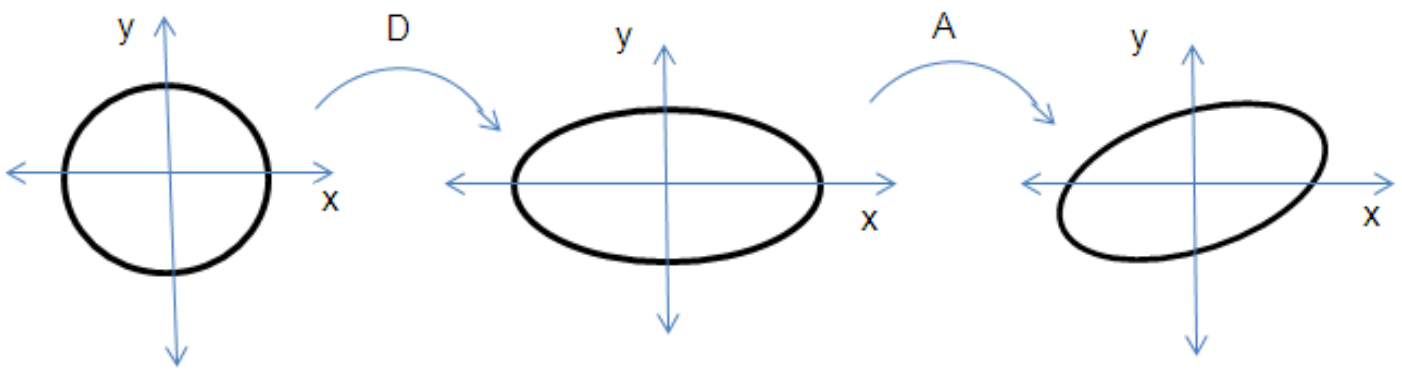

Above discussion extended to in general mostly. If A has full rank now the singular value of A are the lengths of the $\mathrm{n}$ principle semi axis of the ellipsoid the length are $\sigma_{1} \sigma_{2} \ldots \sigma_{n}$ the $\mathrm{n}$ left singular vector of the $\mathrm{A}$ are the directions $u_{1}, u_{2}, \ldots u_{n}$ aligned the $\mathrm{n}$ semi axis of ellipsoid. The $\mathrm{n}$ right singular vectors of $\mathrm{A}$ are the directions $v_{1}, v_{2}, \ldots v_{n}$ in sphere which the matrix $\mathrm{A}$ is transformed into the semi axis of the ellipsoid.

A rank deficient matrix is one whose range is a sub space of $R^{3}$ so it maps the sphere to flat ellipse rather than ellipsoid. 
Let $A=\left[\begin{array}{lll}u_{1} & u_{2} & u_{3}\end{array}\right]\left[\begin{array}{ccc}\sigma_{1} & 0 & 0 \\ 0 & \sigma_{2} & 0 \\ 0 & 0 & 0\end{array}\right]\left[\begin{array}{c}v_{1}^{T} \\ v_{2}^{T} \\ v_{3}^{T}\end{array}\right]$

Here one of the singular value is zero, $u_{3}$ is normal to the ellipse.

Let $V^{T} v_{3}=\left[\begin{array}{l}0 \\ 0 \\ 1\end{array}\right]$ and $D\left[\begin{array}{l}0 \\ 0 \\ 1\end{array}\right]=\left[\begin{array}{l}0 \\ 0 \\ 0\end{array}\right]$

\section{APPLiCATion}

\subsection{Rank- deficient least square}

The linear system is $A x=y$

$$
\begin{gathered}
\quad x=A^{-1} y \\
x=\left(U D V^{T}\right)^{-1} y \\
x=\left(V^{T}\right)^{-1} D^{-1} U^{-1} y \\
x=\left(V^{-1}\right)^{T} D^{-1} U^{-1} y[\because U \text { is orthogonal }] \\
\quad X=\left(V D^{-1} U^{T}\right) b \\
\text { Where } D^{-1}=\operatorname{diag}\left(\frac{1}{\sigma_{1}}, \frac{1}{\sigma_{2}}, \ldots, \frac{1}{\sigma_{n}}\right)
\end{gathered}
$$

The above process is simplest but not fastest. The process is facts if the singular values are zero. If the matrix A is singular the this work.

\section{How to solve}

A is a $n \times n$ square matrix

And rank of $\mathrm{A}$ is $r>n$

The system will not have unique solution if $A x^{*}-y$ is minimum

$A\left(x^{*}+b\right)-y$ for any vector $b \in$ null $(\mathrm{A})[\mathrm{Ab}=0]$

This system is like over and in determined SVD we can solve easily.

First we define residual. Sum of the square of the residual is minimum by least square method. Now to minimize the SVD of A and if then transfer it by $U^{T}$

$$
\begin{gathered}
\|A x-y\|^{2}=\left\|U D V^{T} x-y\right\|^{2} \\
=\left\|I U D V^{T} x-I y\right\|^{2} \\
=\left\|U U^{T} U D V^{T} x-U U^{T} y\right\|^{2} \\
=\|U\|\left\|I D V^{T} x-U^{T} y\right\|^{2} \\
=\left\|D V^{T} x-U^{T} y\right\|^{2} \\
=\left\|\left[\begin{array}{ll}
D & 0 \\
0 & 0
\end{array}\right] V^{T}-\left[\begin{array}{l}
u_{1}^{T} \\
u_{2}^{T}
\end{array}\right] y\right\|^{2} \\
=\left\|\left[\begin{array}{ll}
D & 0
\end{array}\right] V^{T} x-u_{1}^{T} y\right\|^{2}+\left\|u_{2}^{T} y\right\|^{2}
\end{gathered}
$$

the first term can be made zero. The second term is residual

if we let $b=v^{T} x$ and $k=u_{1}^{T} y$

Than split b into $\left[\begin{array}{l}b_{1} \\ b_{2}\end{array}\right]$ 


$$
\begin{gathered}
\therefore\left[\begin{array}{ll}
D_{1} & 0
\end{array}\right]\left[\begin{array}{l}
b_{1} \\
b_{2}
\end{array}\right]=k \\
D_{1} b_{1}=k
\end{gathered}
$$

Now we will go for the minimizing solution than $x=V_{1} b_{1}$

Briefly $x=V D_{1}^{-1} k$

$$
x=V_{1} D_{1}^{-1} U_{1}^{T} y
$$

\section{Conclusion}

This paper discussed about geometric interpretation of SVD and solution of linear system with rank deficiency. This work can be extended to full rank of the matrix A and constructing least square problem.

\section{REFERENCES}

[1] Gary E.Adams, Adam W.Bojanzcyk, and FranklinT. Luk, Computing the PSVD oftwo $2 \times 2$ triangular matrices, SIAM J.Matrix Anal. Appl. vol15,no2,pp366-382,April1994.

[2] Harry C.Andrews and Claude L.Patterson, Outer Product Expansions and their uses in digital image processing, The Amer MathMonthly, pp1-13,January1975.

[3] S.J. Blank, Nishan Krikorian, and David Spring, Ageometrically inspired proofofthe singular valuede composition, The Amer MathMonthly, pp238-239, March 1989.

[4] Adam Bojanczyk and Paul VanDooren, On propagating orthogonal transformations in a product of $2 \times 2$ triangular matrices, in Lothar Reichel, Arden Ruttan, and Richard S.Varga, Eds. Nu- merical Linear Algebra: Proceedings of the Conference in Numerical Linear Algebraand Scientific Computation, Kent (Ohio), March 13-14, 1992, pp1-9, W.deGruyter, NewYork, 1993.

[5] Randall E.Cline, Elements of the theory of generalized inverses formatrices. Education Develop- ment Center, Newton, Mass,1979.

Citation: J. Raghavendar, V. Dharmaiah., "Geometrical Interpretation of Singular Value Decomposition (Svd) \& Applications of SVD ", International Journal of Scientific and Innovative Mathematical Research, vol. 5, no. 4, p. 4, 2017., http://dx.doi.org/10.20431/2347-3142.0504004

Copyright: (C) 2017 Authors. This is an open-access article distributed under the terms of the Creative Commons Attribution License, which permits unrestricted use, distribution, and reproduction in any medium, provided the original author and source are credited. 